\title{
Correspondence
}

\section{On the Variable Capacity Property of CC/DS-CDMA Systems}

\author{
Li-Peng Wang, Yang Yang, Hsiao-Hwa Chen, and Yong-Hua Song
}

\begin{abstract}
A complete complementary code based direct sequence code division multiple access (CC/DS-CDMA) system has been proposed recently as a potential candidate for beyond third generation (B3G) wireless communications. This paper addresses the issues that design of efficient code assignment schemes should be based on a flexible physical layer support, which is extremely important for emerging cross-layer designs in future wireless applications. The study in this paper considers a CC/DS-CDMA system with multiple time slots, three traffic classes and two dynamic code-flock assignment schemes, namely random assignment (RA) and compact assignment (CA). Simulation results show that the CC/DS-CDMA system has variable capacity property (VCP), which is sensitively affected by different code-flock assignment schemes. In general, CA can offer lower blocking probability, whereas RA can offer a larger mean system capacity and higher throughput when offered traffic is heavy.
\end{abstract}

Index Terms-Code division multiple access (CDMA), complete complementary codes, resource allocation.

\section{INTRODUCTION}

The third generation ( $3 \mathrm{G})$ mobile communication systems, such as wideband code division multiple access (WCDMA) and CDMA 2000, have been deployed in many countries for commercial services. In the past several years, we have witnessed a rapid growth of various data-centric wireless multimedia applications, e.g., short message service (SMS), multimedia message service (MMS), web browsing, video telephony, interactive games, traffic navigation, etc. These popular wireless applications and services have greatly enriched our experiences of using mobile phones. It can be envisaged that future beyond $3 \mathrm{G}(\mathrm{B} 3 \mathrm{G})$ mobile communication systems could provide much higher data transmission rates (e.g., $100 \mathrm{Mb} / \mathrm{s}$ ) to support more diverse broadband multimedia applications such as high-quality real-time video streaming and conferencing. However, the existing CDMA technologies [1] can hardly offer high spectrum efficiency and low system complexity, which are required by future B3G broadband wireless communications. This is because all traditional CDMA systems suffer a serious performance bottleneck stemmed from their interference-limited capacity, which significantly reduces the number of simultaneous mobile users in the same system [2]. To overcome the interference-limited problem in all current CDMA systems, innovation in air-interface technologies is the key to ensure broadband quality of service $(\mathrm{Q} O \mathrm{~S})$ required in future B3G mobile communication services.

Based on complete complementary (CC) codes [3]-[5] two novel CDMA systems, namely "CC-code-based offset-stacked spreading

Manuscript received October 16, 2005; revised November 9, 2005. The review of this paper was coordinated by Prof. X. Shen.

L.-P. Wang and Y.-H. Song are with School of Engineering and Design, Brunel University, London UB8 3PH, U.K. (e-mail: lipeng.wang@ brunel. ac.uk; y.h.song@brunel.ac.uk).

Y. Yang is with the Department of Electronic and Electrical Engineering, University College London, London WC1E 6BT, U.K. (e-mail: y.yang@ee.ucl.ac.uk).

H.-H. Chen is with the Institute of Communications Engineering, National Sun Yat-Sen University, Kaohsiung, Taiwan, R.O.C. (e-mail: hshwchen@mail. nsysu.edu.tw).

Digital Object Identifier 10.1109/TVT.2006.873817
CDMA" (CC/OS-CDMA) [6] and "CC-code-based direct-sequence spreading CDMA" (CC/DS-CDMA) [7], have been proposed recently for possible applications in $\mathrm{B} 3 \mathrm{G}$ mobile communications. Compared with traditional CDMA systems, CC-code-based CDMA systems use a flock of $\mathrm{CC}$ codes (instead of a single spreading code) to separate distinct traffic channels. Specifically, CC/OS-CDMA combines CC codes with the offset-stacked spreading technique and can achieve multiple access interference (MAI) free radio transmissions. It also provides the capability to dynamically change data transmission rate "on the fly" by simply shifting a number of chips between two neighboring offset stacked bits without affecting other users' transmission modes. However, CC/OS-CDMA cannot completely eliminate multipath interference (MI) so that it is still a self-interfered system. As a special case of CC/OS-CDMA, CC/DS-CDMA scheme is realized by setting the relative chip shift (offset) between two consecutive bits equal to the length of an element $\mathrm{CC}$ code.

As its name implies, the CC/DS-CDMA scheme combines CC codes with the direct-sequence spreading technique to achieve isotropic MAI- and MI-free radio transmissions [7]. Each mobile terminal, depending on its specific bandwidth (or data rate) requirement, is assigned a flock of $k$ element $\mathrm{CC}$ codes, which should be transmitted simultaneously but separately by $k$ frequency carriers. Therefore, CC/DS-CDMA systems adopt a multicarrier technique to convert a wideband frequency-selective radio channel into a parallel collection of narrowband flat subchannels, and each of them is relatively robust against multipath fading. Each information bit is spread simultaneously by those $k$ element $\mathrm{CC}$ codes assigned to the same user. The bit streams after spreading are then modulated and transmitted in parallel through the corresponding frequency carriers. At the receiver side, a bank of $k$ correlators will perform matched filtering for those element codes received from different frequency carriers, so that an autocorrelation peak can be generated for detection of the transmitted information bit. Mobile users with the same bandwidth requirement can share a common set of frequency carriers for simultaneous radio transmissions using different CC code flocks with the same length as their signatures. Compared with traditional DS-CDMA systems, CC/DS-CDMA has the following advantages: 1) It offers isotropic MAI- and MI-free operations so that the system capacity is greatly improved and becomes virtually noise limited; 2) it has an attractive near-far resistance so that a precise power-control technique is not required; 3) a RAKE receiver, though still improving performance, is no longer a necessity; and 4) other complicated subsystems, such as smart antenna and multiuser detection, which are used in most current CDMA systems, are not necessary. Therefore, CC/DS-CDMA systems can offer a good performance in terms of system capacity, spectrum efficiency, and QoS-enabling capability at a relatively low complexity.

In this paper, we will study the "variable capacity property" (VCP) of the CC/DS-CDMA systems, which we discovered recently and can exert an adverse impact on the system performance. Possible solutions for stabilizing the system capacity will be given and their effectiveness will be discussed. Extensive computer simulations have been used to investigate the behaviors of two proposed resource allocation schemes, namely random assignment (RA) and compact assignment (CA), by taking into account multiple time slots and three traffic classes.

The rest of this paper can be outlined as follows. In Section II, the CC code structure and VCP of CC/DS-CDMA systems are introduced 
TABLE I

EXAMPle of COMPlete COMPLEMENTARy CODES, TyPes 1 AND 2

\begin{tabular}{|c|c|c|}
\hline Code Length & Code Flock & CC Codes \\
\hline \multirow[t]{4}{*}{$L=4$} & \multirow[t]{2}{*}{1} & ++++- \\
\hline & & +-++ \\
\hline & \multirow[t]{2}{*}{2} & ++-+ \\
\hline & & +--- \\
\hline \multirow[t]{16}{*}{$L=16$} & \multirow[t]{4}{*}{1} & +++++-+-++--+--+ \\
\hline & & ++-+-+++++--+++-- \\
\hline & & ++--+--++++++-+- \\
\hline & & +--+++--+-+-++++ \\
\hline & \multirow[t]{4}{*}{2} & ++++-+-+++---++- \\
\hline & & +++-----+--+--++ \\
\hline & & ++---++-++++-+-+ \\
\hline & & ++--+-+++-+----- \\
\hline & \multirow[t]{4}{*}{3} & +++++-+---++-++- \\
\hline & & +-+-++++-++---++ \\
\hline & & ++--+--+----+-+ \\
\hline & & ++-+++---+-+---- \\
\hline & \multirow[t]{4}{*}{4} & ++++-+-+--+++--+ \\
\hline & & +++------++-++-- \\
\hline & & ++---++-----+-+- \\
\hline & & +--+--++-+-+++++ \\
\hline
\end{tabular}

and discussed. Simulation results for a CC/DS-CDMA system with multiple time slots are analyzed in Section III, followed by conclusions in Section IV.

\section{VARIABLE CAPACITY PROPERTY(VCP)}

Let us consider a CC/DS-CDMA system with $C$ users (which can also be called the capacity of the system). Each user in the system is assigned a flock of $\mathrm{CC}$ codes for user separation. Assume that there are in total $n$ frequency carriers available in the system. The CC codes used in such a CC/DS-CDMA system can be of different lengths and can be organized into a hierarchical structure. Specifically, there are $l$ element $\mathrm{CC}$ codes with the same length $l$ bits and they are sequentially denoted by $(l, m)$, where $m=1,2,3, \ldots, l$. Due to the inherent property of CC codes [6], the value of $l$ should be a power of four, i.e., $l=4,16,64,256, \ldots, l_{\max }$. These $l$ codes with the same length are divided into $\sqrt{l}$ code flocks, each contains $\sqrt{l}$ element codes. Therefore, code $(l, m)$ belongs to the $f$ th $(f=\lceil m / \sqrt{l}\rceil)$ code flock, where $\lceil\cdot\rceil$ denotes the ceiling function. Table I shows the hierarchical structure of $\mathrm{CC}$ codes at different lengths, i.e., $l=4,16$.

Code flocks with the same length have the perfect properties of zero cross-correlation and zero out-of-phase autocorrelation. The major difference between CC/DS-CDMA systems and traditional CDMA systems (e.g., IS-95, W-CDMA, and CDMA2000) is that a code flock, instead of a single spreading code, will be used to characterize a traffic channel in CC/DS-CDMA. In doing so, CC/DS-CDMA can achieve MAI- and MI-free radio transmissions. The number of mobile users that can be accommodated in a CC/DS-CDMA system becomes noise limited, rather than interference limited as in traditional CDMA systems. The $l$ element $\mathrm{CC}$ codes with the same length can support up to $f_{\max }=\sqrt{l}$ mobile users with the same bandwidth (or data rate) requirement. The overall processing gain, or spreading factor, of an $l$-bit code flock is calculated by $l \cdot \sqrt{l}$. The $\sqrt{l}$ element codes belonging to the same code flock are assigned to a single user and need to be transmitted simultaneously through independent frequency carriers, i.e., the multicarrier technique should be used in CC/DS-CDMA. For example, the four element $\mathrm{CC}$ codes with length $l=4$ in Table I are divided into two flocks and can support two mobile users; each of them uses a flock of two CC codes and therefore requires two frequency carriers for carrying the code transmissions.

As code flocks with different lengths are usually not orthogonal to each other, they should be separated by independent frequency carriers when they are used in the same CC/DS-CDMA system. For example, code flocks $\{(4,1),(4,2)\}$ and $\{(4,3),(4,4)\}$ are orthogonal to each other so that they can share a common set of two frequency carriers, while code flock $\{(16,1),(16,2),(16,3),(16,4)\}$, requiring four frequency carriers, is not orthogonal to $\{(4,1),(4,2)\}$. To avoid interference, code flocks with different lengths should be transmitted through independent carrier flocks.

Given the number of frequency carriers, say $n\left(n \geq \sqrt{l_{\max }}\right)$, the corresponding CC/DS-CDMA system can support a fixed number of $n\left(=n / \sqrt{l} \cdot f_{\max }\right)$ mobile users, no matter what kinds of code flocks are used. However, when code flocks with different lengths coexist in the same system, the system capacity in terms of data rate will dynamically change according to the occupancy status of frequency carriers. Let $n_{l}$ denote the number of carriers already assigned for carrying $l$-bit code flocks and let $n_{0}$ denote the number of remaining idle carriers. Obviously, we have $n=n_{0}+\sum_{l} n_{l}$. Assuming that all $n_{0}$ idle carriers are used to carry the shortest code flocks (i.e., $l_{\text {min }}=4$ ), system capacity $C$, defined as the maximally achievable data rate at the current occupancy status, is given by

$$
\begin{aligned}
C=\frac{n_{0}}{2} \frac{l_{\max }}{4} \cdot 2 R+\sum_{l} \frac{n_{l}}{\sqrt{l}} \frac{l_{\max }}{l} \cdot \sqrt{l} R \\
=l_{\max } \cdot R \cdot\left(\frac{n_{0}}{4}+\sum_{l} \frac{n_{l}}{l}\right), \\
\quad l=4,16,64, \ldots, l_{\max }
\end{aligned}
$$

where $R$ is the basic data rate supported by the longest code flocks (with code length $l_{\max }$ ). For the extreme case when all $n$ frequency carriers are assigned to the shortest code flocks (i.e., $n_{4}=n$ ), the system capacity achieves the maximum value $C_{\max }=l_{\max } \cdot n \cdot R / 4$. On the other hand, when $n_{l_{\max }}=n$, the minimum system capacity is derived as $C_{\text {min }}=n \cdot R$. In summary, for a CC/DS-CDMA system adopting different-length $\mathrm{CC}$ codes, the system capacity will dynamically change at every instance when the assignment or release of a whole carrier flock occurs. This phenomenon is defined as the VCP of CC/DS-CDMA systems.

Three possible solutions for stabilizing the system capacity are the following.

1) Besides frequency carriers, use the time domain as an additional dimension to separate $\mathrm{CC}$ codes with different lengths.

2) Fix the length of $\mathrm{CC}$ codes, and assign multiple code flocks to a single user for supporting multirate multimedia applications.

3) Design new sets of CC codes so that the code flocks of different lengths will be orthogonal to each other.

Solution 2 looks straightforward but is not efficient when the required data rate is much larger than the basic data rate supported by a single code flock. If realized, solution 3 would be the best choice with high flexibility and efficiency. Our focus in this paper is to evaluate how solution 1 could alleviate VCP in a CC/DS-CDMA system with multiple time slots.

Fig. 1 shows an example CC/DS-CDMA system with three time slots, four frequency carriers $(n=4)$, and two kinds of CC codes with different lengths $(l=4,16)$. Carrier flocks 1 and 2 contain, respectively, two and four frequency carriers in different time slots. In the notation " $2: 4$," the first digit " 2 " denotes the number of existing calls supported by carrier flock 2 , and the second digit " 4 " denotes the capability of this carrier flock. Thus, carrier flock 2 can accommodate two more calls with bandwidth requirement $R$. Conversely, with notation " $2: 2$," carrier flock 1 has reached its maximum capability of carrying two $4 R$ calls. The total capacity of this system, calculated by summing up the capacities in three time slots, is varying in the range 


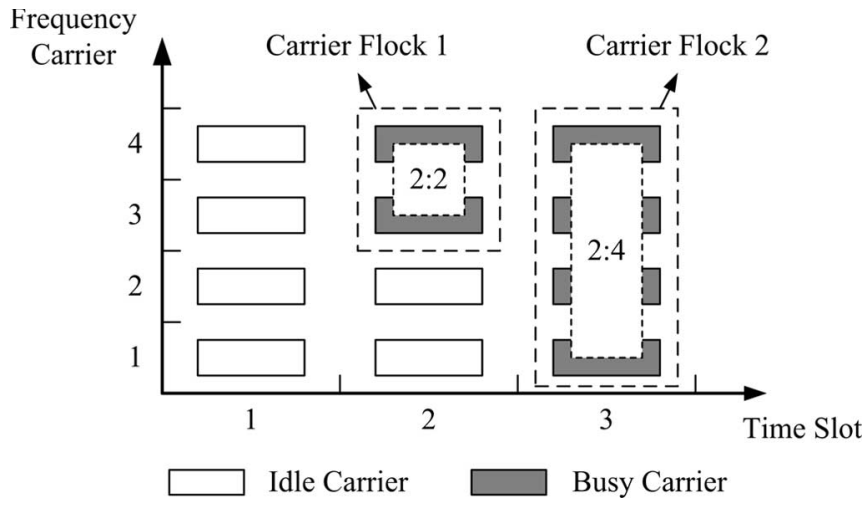

Fig. 1. A CC/DS-CDMA system with three time slots, four frequency carriers $(n=4)$, and two kinds of CC codes $(l=4,16)$.

of $\left[C_{\min }=12 R, C_{\max }=48 R\right]$ and, specifically, is equal to $36 R$ for the carrier/slot occupancy status shown in Fig. 1.

\section{Simulation Results}

In this section, $\mathrm{VCP}$ is investigated through extensive computer simulations. The CC/DS-CDMA system under consideration consists of multiple CC code flocks with three different lengths $(l=4,16,64)$, 32 frequency carriers $(n=32)$, and five time slots (for uplink or downlink traffic).

Wireless traffic is assumed as a mix of three classes of applications, which are generated at different Poisson arrival rates and have different exponentially distributed service times. The offered traffic (in erlangs), defined as the multiplication of arrival rate and average service time, of different application classes are in the ratio $G_{1}: G_{2}: G_{3}=4: 2: 1$. The corresponding bandwidth (or data rate) requirements of these three traffic classes are $R, 4 R$, and $16 R$, which are supported by CC code flocks with lengths 64,16 , and 4 , respectively. This traffic model simulates the situation that low-bandwidth calls occur more frequently than high-bandwidth ones in the system. More traffic classes with other bandwidth requirements can be supported by assigning multiple code flocks to a single user. This paper focuses on the single code-flock assignment and transmission case.

For the purpose of comparison, two code-flock assignment schemes, namely random assignment (RA) and compact assignment (CA), are used in the investigation of VCP. Upon receiving a new call request, the base station first identifies all suitable frequency carriers in each time slot according to this call's bandwidth requirement. Note that only the calls with the same bandwidth requirement can share a common carrier flock (by using different code flocks with the same length) without generating interference to each other. As its name implies, the RA scheme will select at random a suitable flock of frequency carriers for accommodating the new call, while the CA scheme will first check the existing carrier flocks that are carrying the same traffic class. If all such carrier flocks are full so that it cannot accommodate this call, a new carrier flock in the busiest time slot (with sufficient idle frequency carriers) will be formed to carry the new call. In other words, the new call assignment under $\mathrm{CA}$ is packed as tightly as possible into the existing carrier flocks or busy time slots. The remaining resources (frequency carriers and time slots) are therefore maximally aggregated and very flexible for supporting different traffic classes. As an example, consider the CC/DS-CDMA system shown in Fig. 1. Let the notation " $x . y$ " denote the $y$ th frequency carrier in time slot $x$. The existing carrier flocks 1 and 2 can then be represented by $(2.1,2.2)$ and $(3.1,3.2,3.3,3.4)$, respectively. For a new call with bandwidth requirement $R$, there are two suitable carrier flocks for selection, i.e.,

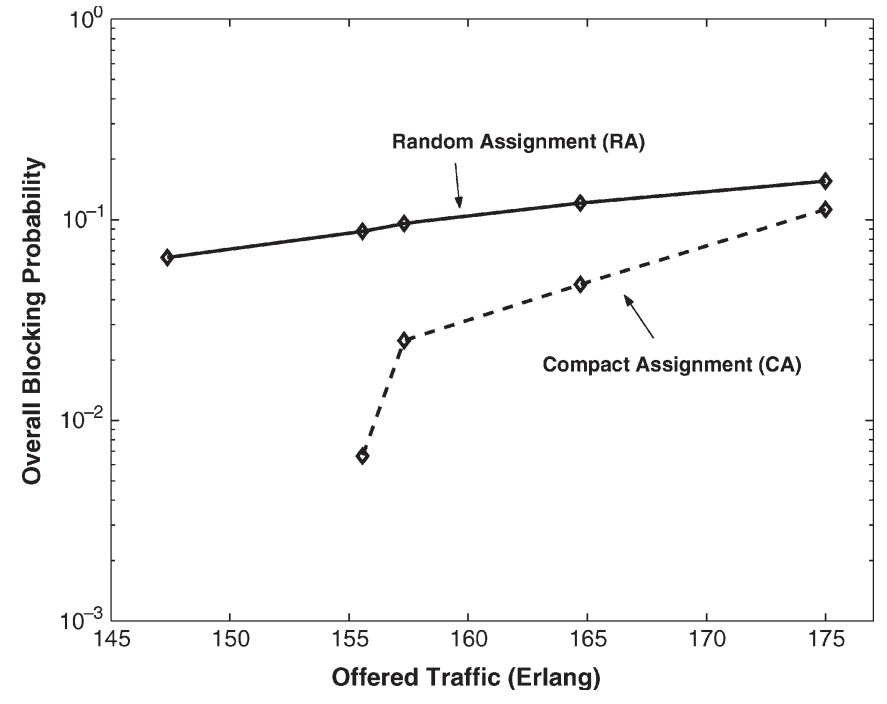

Fig. 2. Overall blocking probability.

$\{(1.1,1.2,1.3,1.4),(3.1,3.2,3.3,3.4)\}$. The RA scheme will select at random from these two choices, while the CA scheme will select (3.1, $3.2,3.3,3.4$ ) to let the new call share these four frequency carriers with two ongoing calls with the same bandwidth requirement. For a new call with bandwidth requirement $4 R$, three suitable carrier flocks can be identified by the base station, i.e., $\{(1.1,1.2),(1.3,1.4),(2.3,2.4)\} .{ }^{1}$ As usual, the RA scheme will randomly select one carrier flock from these three. However, the CA scheme will select $(2.3,2.4)$ because these two frequency carriers are in the busy time slot 2 . In doing so, all frequency carriers in time slot 1 are kept idle and flexible for supporting future new calls with different bandwidth requirements.

The simulation results shown in the following figures are obtained from the experiments of at least 300000 calls for each offered-traffic value. Fig. 2 shows the overall blocking probability as a function of offered traffic under the RA and CA schemes. As expected, CA offers better blocking probability performance than RA over the entire loading range. This is because CA has the capability of aggregating idle frequency carriers together, so that more low-bandwidth calls requiring/sharing large carrier flocks can be accommodated. On the other hand, the RA scheme favors high-bandwidth calls requiring small carrier flocks, especially when offered traffic is heavy. Figs. 3 and 4 show the VCP under the CA and RA schemes, respectively. For the purpose of comparison, total offered traffic $G$ in both simulation experiments is set to be $G=G_{1}+G_{2}+G_{3}=155$ (erlangs). The system capacity, calculated by (1) in each time slot, is updated at every assignment or release instance of a whole carrier flock. The corresponding capacity curves (in unit of basic data rate $R$ ) at two different time scales are given as parts (a) and (b) in those figures. After a 2000-s initialization period, CA (Fig. 3) offers a much smaller variation in system capacity than RA (Fig. 4). The large capacity variation is due to the assignments or releases of large carrier flocks, which can accommodate many low-bandwidth calls. Note that the CA scheme always tries to use the existing carrier flocks to support new calls in the same class. Therefore, compared with RA, it is more difficult to empty and release a carrier flock under CA, especially when the carrier flock is large and offered traffic is heavy.

At different offered-traffic values, the standard deviations and the means of system capacity (in unit of $R$ ) under the CA and RA schemes

${ }^{1}$ Carrier flocks $\{(1.1,1.2),(1.3,1.4)\}$ can be replaced by $\{(1.1,1.3),(1.2$, $1.4)\}$ or $\{(1.1,1.4),(1.2,1.3)\}$. 


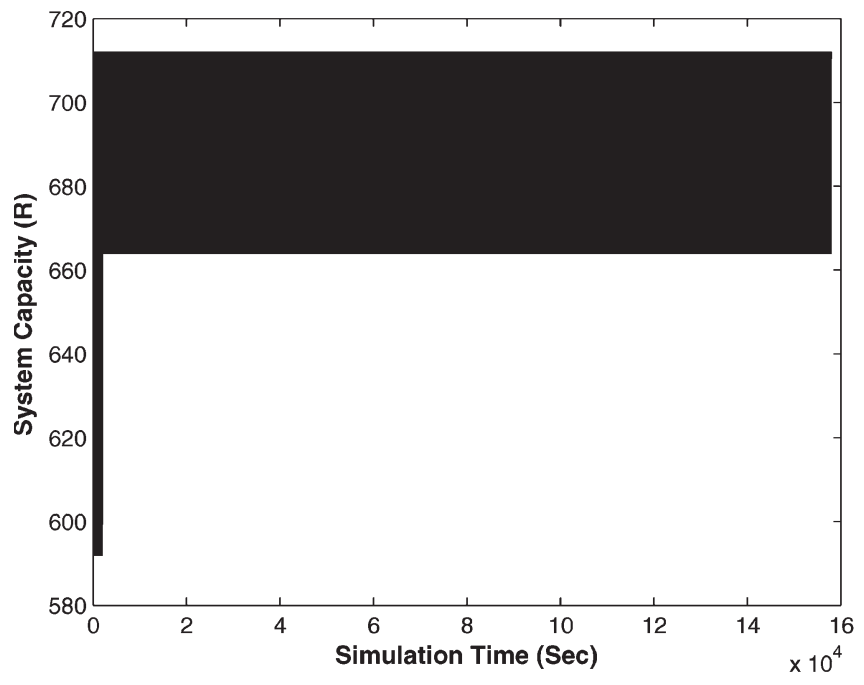

(a)

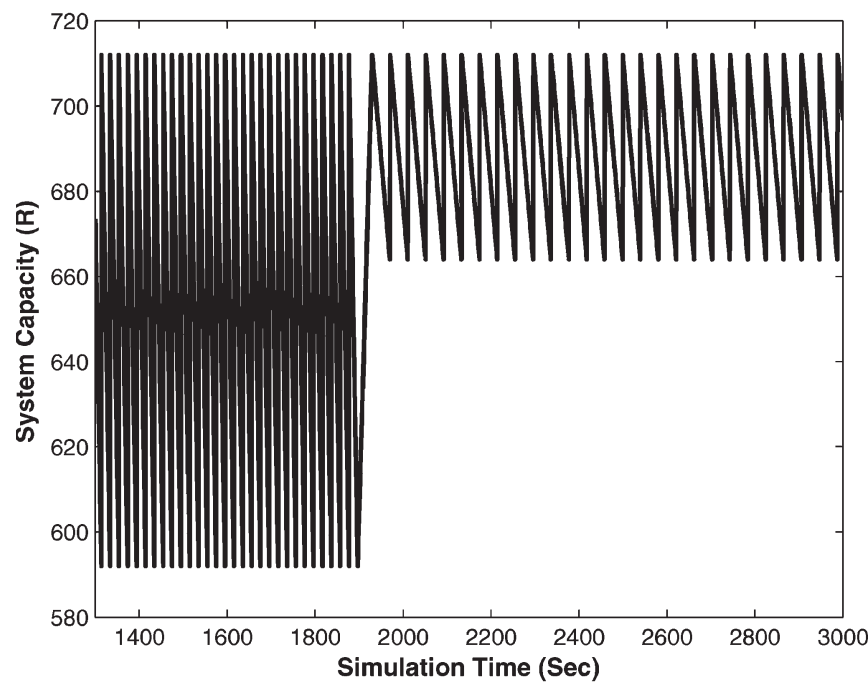

(b)

Fig. 3. System capacity under the CA scheme, offered traffic $G=G_{1}+$ $G_{2}+G_{3}=155$ erlangs

are compared in Figs. 5 and 6, respectively. As seen in Fig. 5, CA is much better than RA in keeping the variance of system capacity small.

Regarding the mean of system capacity, the results shown in Fig. 6 look surprising. Here, we use circle symbols to mark the mean systemcapacity curves and triangle symbols for the average throughput curves. When offered traffic is light, say $G=155$ erlangs, the system capacities under the CA and RA schemes are comparable because most new calls can be accommodated by the system. By packing new calls tightly with the existing calls, the CA scheme aggregates idle frequency carriers together so that it is more flexible to support new calls with different bandwidth requirements, especially those low-bandwidth calls requiring large carrier flocks. As a result, CA can offer lower blocking probability (Fig. 2) and higher throughput (Fig. 6) than RA at light offered traffic. On the other hand, when offered traffic is heavy, RA can offer much larger system capacity and higher throughput than $\mathrm{CA}$, although the resource utilization (ratio between throughput and capacity) under RA is much poorer. This interesting result is due to the fact that the releases of carrier flocks are much less frequent in CA than in RA. After the initialization period, the CA scheme divides the system resources, i.e., frequency carriers and time slots, to different traffic classes. Many calls with the

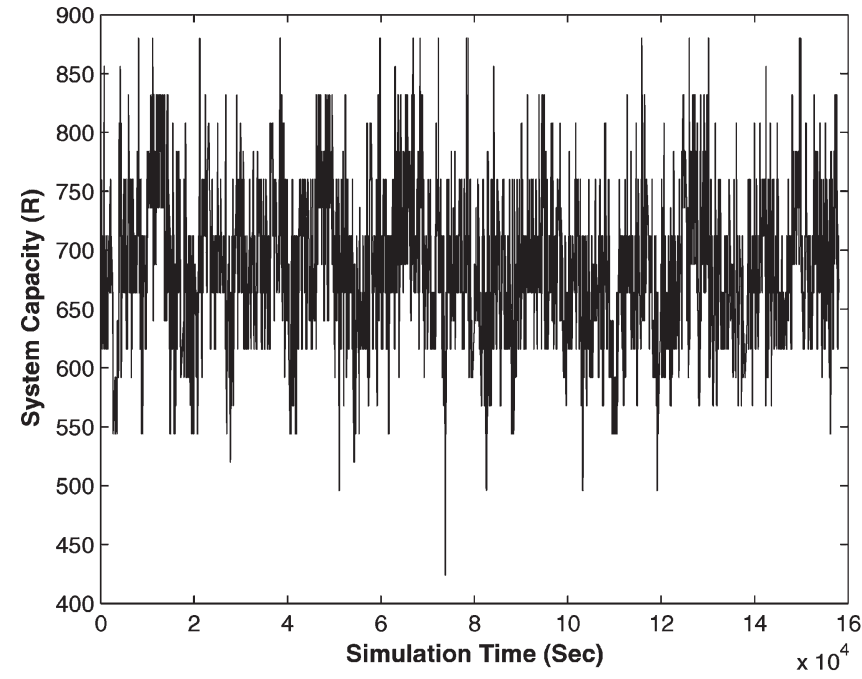

(a)

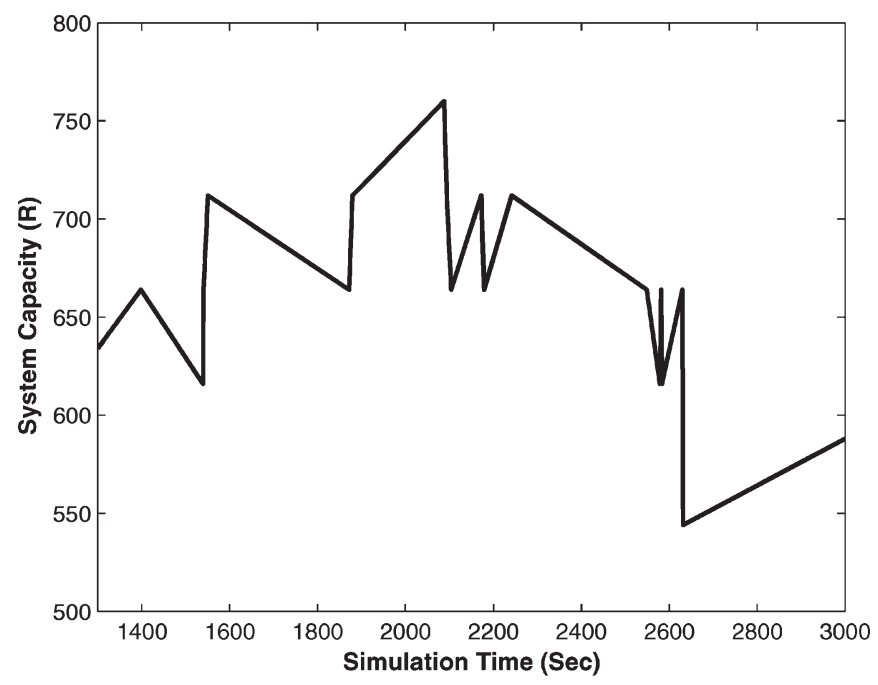

(b)

Fig. 4. System capacity under the RA scheme, offered traffic $G=G_{1}+$ $G_{2}+G_{3}=155$ erlangs.

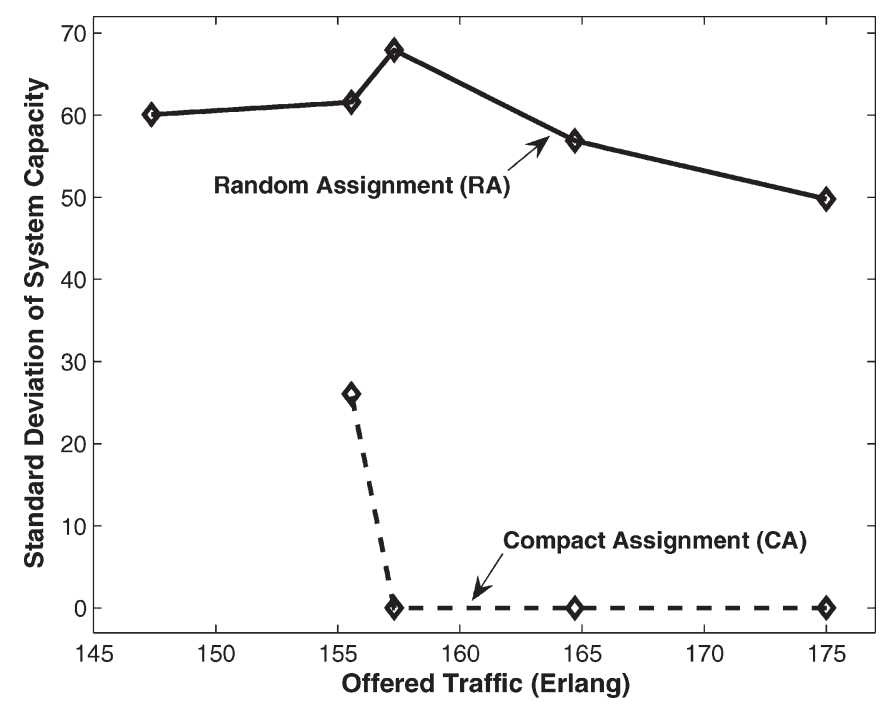

Fig. 5. Standard deviation of system capacity. 


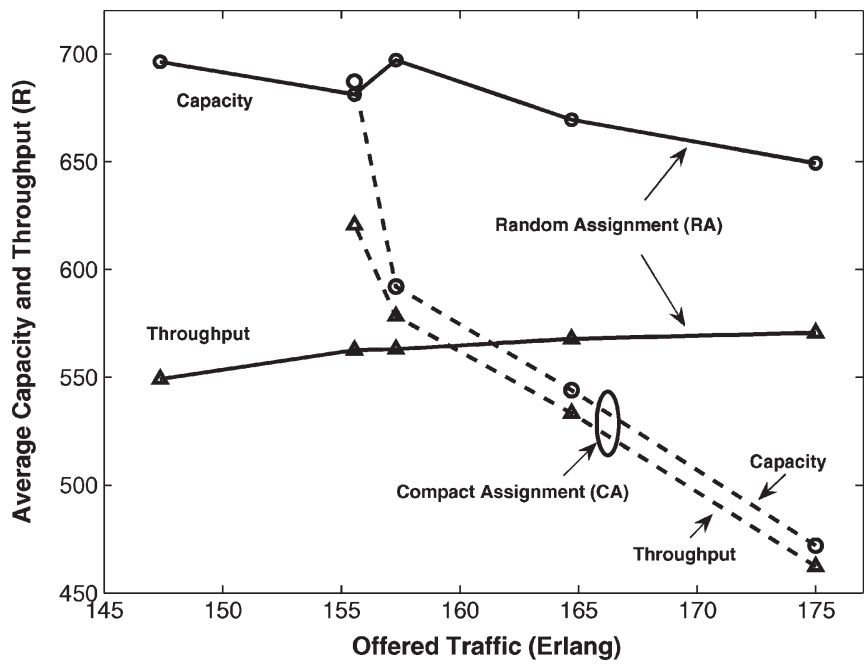

Fig. 6. Average system capacity and throughput.

same bandwidth requirement are sharing common carrier flocks or, in other words, kept in a compact state. Carrier flocks, once assigned by $\mathrm{CA}$ to specific traffic classes, are unlikely to become empty and then be released, especially when offered traffic is heavy and a largesize carrier flock supporting many low-bandwidth calls is considered. Compared with CA, the RA scheme cannot manage system resources very well. Frequency carriers are often segmented by RA into small flocks and new calls are not required to share carrier flocks with the existing calls. As a result, this difference in average size of carrier flocks between RA and CA increases in proportion to the offered traffic. As the assignments and releases of small carrier flocks are much easier and more frequent in RA, the variation of system capacity under RA is much larger than that under CA (Fig. 5). In addition, since RA generates more small carrier flocks to carry high-bandwidth calls, it can offer a larger mean system capacity and a higher throughput than CA at heavy offered traffic (Fig. 6).

\section{CONCLUSION}

Through extensive computer simulations, we have investigated the VCP of a CC/DS-CDMA system with multiple time slots. The block- ing probability, variable system capacity, and throughput performance under two different code-flock assignment schemes have been derived and compared. The great flexibility in supporting variable data rates, QoS requirements, etc., makes CC/DS-CDMA an important candidate for future wireless systems based on the cross-layer optimized design. It is found that the current CC/DS-CDMA system can support a constant number of users/calls, irrespective of their different bandwidth requirements. However, the system capacity in terms of data rate can vary with instantaneous traffic and system resources utilization (i.e., carrier frequencies and time slots). Our study has also revealed that the VCP of the CC/DS-CDMA systems can be affected sensitively by different code assignment schemes. To remove the VCP completely, we should generate some new CC code sets that can preserve the orthogonality among the code flocks of different lengths.

\section{ACKNOWLEDGMENT}

The authors would like to thank Associate Editor Prof. X. Shen and anonymous reviewers for their useful comments on the preliminary version of this paper.

\section{REFERENCES}

[1] T. Ojanpera and R. Prasad, "An overview of air interface multiple access for IMT-2000/UMTS," IEEE Commun. Mag., vol. 36, no. 9, pp. 82-95, Sep. 1998.

[2] A. M. Viterbi and A. J. Viterbi, "Erlang capacity of a power controlled CDMA system," IEEE J. Sel. Areas Commun., vol. 11, no. 6, pp. 892-900, Aug. 1993.

[3] M. Golay, "Complementary series," IRE Trans. Inf. Theory, vol. II-7, pp. 82-87, Apr. 1961.

[4] N. Suehiro, "Complete complementary code composed of n-multiple-shift orthogonal sequences," Trans. IEICE Jpn., vol. J65-A, pp. 1247-1253, Dec. 1982 (in Japanese).

[5] N. Suehiro and M. Hatori, "N-shift cross-orthogonal sequences," IEEE Trans. Inf. Theory, vol. 34, no. 1, pp. 143-146, Jan. 1988.

[6] H.-H. Chen, J.-F. Yeh, and N. Suehiro, "A multicarrier CDMA architecture based on orthogonal complementary codes for new generations of wideband wireless communications," IEEE Commun. Mag., vol. 39, no. 10, pp. 126-135, Oct. 2001.

[7] H.-H. Chen, J.-X. Lin, S.-W. Chu, C.-F. Wu, and G.-S. Chen, "Isotropic air-interface technologies for fourth generation wireless communications," Wiley Wirel. Commun. Mob. Comput., vol. 3, no. 6, pp. 687-704, Sep. 2003. 